\title{
Sprechen Männer und Frauen eine andere Sprache? Eine Untersuchung geschlechterspezifischer Unterschiede in der Wortwahl und Syntax am Beispiel der Lifestylemaga- zine Brigitte und Men's Health
}

\author{
Lecturer Veronica CÂMPEAN, PhD \\ Department of Communication, Public Relations and Advertising \\ Faculty of Political, Administrative and Communication Sciences \\ Babeş-Bolyai University, Cluj-Napoca, Romania \\ E-mail: campean@fspac.ro
}

\begin{abstract}
The article Do women and men speak a different language? Gender-specific differences in word-choice and syntax in two lifestyle magazines: Brigitte und Men's Health aims to pinpoint gender-specific differences in the linguistic behaviour of men and women and to determine whether the above-mentioned magazines indeed feature male and female language. The subject is approached from the following perspectives: we begin by analysing the general linguistic features of the articles in the light of female and male specificity, then we trace the way in which the same subject was edited with a different word-choice, only to conclude by filtering out common linguistic tendencies. The analysed corpus includes the first issues of both publications from 2014, 2015 and 2016. Only those categories were selected that offered similar content to both target audiences, even though their title might not be identical.
\end{abstract}

Keywords: female language; male language; gender-specific differences; linguistic tendencies; magazine language. 


\section{Literarturliste:}

1. Ayaß, Ruth (2008). Kommunikation und Geschlecht: Eine Einführung, Stuttgart: W. Kohlhammer.

2. Brielmaier, Peter; Wolf, Eberhard (1997). Zeitungs- und Zeitschriftenlayout, 1., Auflage, Reihe praktischer Journalismus, Band 30, Konstanz: UVK Medienverlag.

3. Câmpian, Veronica (2010). Grundlagen der verbalen und nonverbalen Kommunikation, Cluj-Napoca: Accent.

4. Dudenreihe (2002). Das Bedeutungswörterbuch, Band 10, 3., aktualisierte Auflage, Mannheim: Dudenverlag. 
5. Falgar, Adrianna (2002). Macht und Machtlosigkeit - Frauensprache in der Männerwelt; Fakten, Hintergründe, Konsequenzen, Stuttgart: Heinz.

6. Gottburgsen, Anja (2004). Kleiner Unterschied, große Wirkung: Die Wahrnehmung vom weiblichen und männlichem Kommunikationsverhalten. In: Eichhoff-Cyrus, Karin (Hg.): Adam, Eva und die Sprache - Beiträge zur Geschlechterforschung, Mannheim: Dudenverlag, S. 148-175.

7. Hecker, Alexandra (2013). Soziolinguistik; Kommunikation und Kommunikationsprobleme bei Paaren unterschiedlicher kultureller Herkunft, Hamburg: Diplomica.

8. Rus, Flaviu, Călin; Câmpian, Veronica (2007). Darstellungsformen in den Medien, Mittweida: Hochschulverlag.

9. Trömel-Plötz, Senta (2007). Frauensprache: Sprache der Veränderung, Neuauflage, Frankfurt am Main: Fischer.

\section{Korpus:}

10. Brigitte $1 / 2014$

11. Brigitte $1 / 2015$

12. Brigitte $1 / 2016$

13. Men' Health $1 / 2014$

14. Men' Health 1/2015

15. Men' Health 1/2016 